\title{
G7063-2, A NEW NITROGEN-CONTAINING ANTIBIOTIC OF THE EPOXYDON GROUP, ISOLATED FROM THE FERMENTATION BROTH OF A SPECIES OF STREPTOMYCES
}

\author{
Mary Noble and D. Noble \\ Biochemistry Department, Glaxo Research Limited, Sefton Park, \\ Stoke Poges, Buckinghamshire, England \\ R. B. SYKES \\ Antibiotics Research Department, Glaxo Research Limited, \\ Greenford, Middlesex, England
}

(Received for publication March 22, 1977)

\begin{abstract}
Antibiotic G7063-2, isolated from a Streptomyces species, is a new nitrogen-containing analogue of phyllostine and terreic acid. In vitro G7063-2 is moderately active against both Gram-positive and Gram-negative bacteria and weakly active against fungi. It is toxic to mice.
\end{abstract}

An antibiotic, called G7063-2, was isolated from the culture broth of a species of Streptomyces. Physical and chemical measurements of the crystalline antibiotic suggest it to be 4-amino-7-oxa- bicyclo $[4,1,0]$ hept-3-ene-2,5-dione-3-carboxamide (I). It thus belongs to a quinonelike group of antibiotics referred to as the epoxydon group in the classification of BERDY ${ }^{1)}$.

This paper describes the production, isolation and properties of this antibiotic.

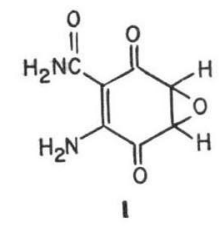

\section{Experimental}

Organism. The antibiotic producing organism is a species of Streptomyces isolated from garden soil at Gothenburg, Sweden. It has been deposited in the National Collection of Industrial Bacteria, Aberdeen, Scotland and designated as NCIB 11306.

Fermentation. The organism was grown on slopes containing malt extract (L39, Oxoid Ltd., London, England), 2.4\%; yeast extract (L21, Oxoid Ltd.,), 0.5\% and agar (no. 3, Oxoid Ltd.,), 1.5\%, made up in tap water and adjusted to $\mathrm{pH} 7.8$ with sodium hydroxide.

Growth from one slope was suspended in $10 \mathrm{ml}$ sterile distilled water and $1 \mathrm{ml}$ of the suspension was transferred to $60 \mathrm{ml}$ of sterilised medium containing soya bean meal (J. Bibby \& Sons Ltd., Liverpool, Lancashire, England), 1.5\%; casein hydrolysate (BDH Chemicals Ltd., Poole, Dorset, England), $0.1 \%$; glycerol (Evans Medical Ltd., Liverpool, Lancashire, England), $2 \%$ and sodium nitrate, $0.3 \%$. The inoculated medium was shaken ( $220 \mathrm{rev} /$ minute on a rotary shaker with a 2 -inch throw) in a $250-\mathrm{ml}$ conical flask for 48 hours at $28^{\circ} \mathrm{C}$.

A portion $(80 \mathrm{ml})$ of the shake flask fermentation was transferred to 4 litres of the same medium in a 5 -litre fermenter. The mixture was stirred ( $550 \mathrm{rev} / \mathrm{minute})$ and aerated ( 3 litres air/minute) for 24 hours at $22^{\circ} \mathrm{C}$.

A portion (3 litres) of the 5-litre fermentation was transferred to 150 litres of the same medium in a 230 -litre fermenter. The mixture was stirred ( $350 \mathrm{rev} /$ minute) and aerated ( 284 litres air/minute) for 48 hours at $22^{\circ} \mathrm{C}$. 
Antibiotic Isolation. Broth (135 litres) at harvest was adjusted to $\mathrm{pH} 7$ with sulphuric acid and centrifuged for a throughput time of 45 minutes (KA6 chamber bowl centrifuge; Westfalia Separator A. G., 4740, Oelde, Westfalia, Germany) to separate aqueous solution from mycelium. The aqueous solution (125 litres) was extracted with 3 one-third volume of ethyl acetate and the combined ethyl acetate extracts were evaporated to dryness.

The residue was extracted with $350 \mathrm{ml} n$-butanol - methanol - water (4:1:2 by volume), filtered to remove inactive solid and the extract was fractionated on a column of Sephadex LH20 $(160 \times 6 \mathrm{~cm}$; Pharmacia Fine Chemicals AB, Uppsala, Sweden) in the same solvent mixture. Fractions active against both Staphylococcus aureus and Escherichia coli were separated from fractions active only against Staphylococcus aureus. The fractions with activity against both organisms were combined and evaporated to dryness.

The residue was extracted with ethyl acetate and filtered. The filtrate was evaporated to $30 \mathrm{ml}$ and applied to a column of Sephadex LH20 $(120 \times 3.5 \mathrm{~cm})$ packed in chloroform - ethyl acetate $(1: 2$ by volume). Elution was with the same solvent and active fractions were combined, evaporated to $70 \mathrm{ml}$ and kept at $-20^{\circ} \mathrm{C}$.

After 3 days yellow crystals were collected, washed with cold ethyl acetate and dried under reduced pressure to give $430 \mathrm{mg}$ solid.

Microbiological Assay. To follow the production of antibiotic during fermentation, samples of broth were centrifuged and portions of the supernatant assayed by an agar diffusion cup-plate method. The agar contained beef extract (L29, Oxoid Ltd.), 0.8\%; bacteriological peptone (L34, Oxoid Ltd.), $1 \%$; sodium chloride, $0.5 \% ; 2,3,5$-triphenyl tetrazolium chloride, $0.008 \%$ and agar (no. 3, Oxoid Ltd.), $1.2 \%$. Test organisms were Staphylococcus aureus Oxford H strain VI and Escherichia coli NCIB 9482. Assay plates were incubated at $37^{\circ} \mathrm{C}$ for 16 hours and the diameter $(\mathrm{mm})$ of zones of growth inhibition recorded.

Isolation of activity was followed by an agar diffusion method in which portions of samples were applied to Whatman 3MM paper (W. \& R. Balston Ltd., Maidstone, Kent, England). The paper was air-dried to remove volatile substances, placed on a 2 -mm layer of solidified agar $(0.7 \%$; no. 3, Oxoid Ltd.) and overlayered with nutrient agar (beef extract, $0.57 \%$; bacteriological peptone, $0.71 \%$; sodium chloride, $0.36 \%$; tetrazolium chloride, $0.006 \%$ and agar, $0.86 \%$ ) and test organism. Assay plates were treated as given for the cup-plate method.

Minimum inhibitory concentrations (MIC) of the antibiotic were determined for various bacteria by a serial dilution method modified from that described by TIPPETT et al. ${ }^{2)} \quad$ Trays (Sterilin Ltd., Teddington, England) that contained thirty-six wells, each of about $1 \mathrm{ml}$ volume were used. Dilutions of the antibiotic were made in nutrient medium containing phenol red indicator (bacteriological peptone, L37, Oxoid Ltd., $1 \%$; beef extract, Lab-Lemco, Oxoid Ltd., $1 \%$; sodium chloride, $0.5 \%$; glucose, $2 \%$ and phenol red, $0.02 \%)$. A portion $(100 \mu \mathrm{l})$ of the solution of test compound at each dilution was added to a well. The same medium $(100 \mu \mathrm{l})$ containing the appropriate organism was then added to give a final inoculum concentration in the well of $10^{5}$ organisms $/ \mathrm{ml}$. The trays were incubated at $37^{\circ} \mathrm{C}$ for 18 hours. The MIC $(\mu \mathrm{g} / \mathrm{ml})$ was taken as the smallest concentration of antibiotic at which the indicator remained red; yellow colour indicated acid production caused by growth of the organism.

Antibiotic activity against a range of fungi was determined by a serial dilution method in tubes. Dilutions of the antibiotic were made in SABOURAUD-maltose medium (mycological peptone, Oxoid L40, $1 \%$; maltose $4 \%$ ) and $2 \mathrm{ml}$ of each dilution added to a tube. Fungal inoculum was prepared by washing, with medium, the growth of the appropriate fungus from an agar slope containing SABOURAUDmaltose medium. A portion $(100 \mu 1)$ of the resultant fungal suspension was added to each tube. Yeast inoculum was prepared by incubating the appropriate yeast at $37^{\circ} \mathrm{C}$ for 6 hours in SABOURAUD-maltose medium then adding $100 \mu \mathrm{l}$ amounts of the culture broth to the appropriate tubes. Tubes containing fungi and yeast were incubated at $27^{\circ} \mathrm{C}$ for 1,2 or 7 days. The $\mathrm{MIC}(\mu \mathrm{g} / \mathrm{ml})$ was taken as the smallest concentration of antibiotic preventing growth of the test organism.

Thin-Layer Chromatography. The isolation of the antibiotic was also followed by TLC. Samples were applied to either layers of cellulose containing fluorescent indicator $(20 \times 20 \mathrm{~cm}$; 13254 ; Eastman- 
Kodak Co., Rochester, N. Y., U. S. A.) or to layers of keiselgel $60 \mathrm{~F} 254(20 \times 20 \mathrm{~cm}$; 5735; E. Merck, Darmstadt, Germany). Development was with butanol - methanol - water (4:1:2 by volume) for cellulose plates and with ethyl acetate for silica plates. Other solvents (Table 1) were also used for the characterisation of the antibiotic. Solvents were either of Analar grade or were redistilled. After development at $24^{\circ} \mathrm{C}$ sheets were air-dried, examined under U. V. light (254 and $\left.356 \mathrm{~nm}\right)$ and overlayered with test organism as described under Microbiological Assay. Rf values of zones of growth inhibition were recorded.

Spectroscopy. The mass spectrum (electron ionisation) was recorded on a Varian MAT 311A spectrometer and the carbon-13 NMR spectrum on a Bruker HX90E spectrometer.

Evaporation. All solutions were evaporated under reduced pressure, large volumes with a potstill, volumes of 20 litres or below with a rotary evaporator.

Acute Mouse Toxicity. Test compound was dissolved in water-methanol (1:1 by volume) and dilutions made with the same solvent. Each concentration of compound was administered i. p. to a group of 5 albino female mice (Charles River, Harefield strain). Each mouse weighed $20 \mathrm{~g}$ and received $0.2 \mathrm{ml}$ of the appropriate test solution. Survivors were counted daily for seven days.

\section{Results}

\section{Physical and Chemical Properties}

G7063-2 is a yellow crystalline antibiotic, soluble in water, methanol, dimethylsulphoxide and acetone and slightly soluble in chloroform. It turns red with the loss of antibiotic activity in sodium carbonate solution at $\mathrm{pH} 10$.

Crystalline material decomposes above $180^{\circ} \mathrm{C}$. The mass spectrum (Fig. 1) shows a base peakmolecular ion at $m / e$ 182; an accurate mass measurement for this peak gave a value of 182.0326 indicating a molecular formula of $\mathrm{C}_{7} \mathrm{H}_{6} \mathrm{~N}_{2} \mathrm{O}_{4}$. Fragmentation peaks at $m / e 137$ and 109 correspond with the loss of $\mathrm{CH}_{3} \mathrm{NO}$ and $\mathrm{C}_{2} \mathrm{H}_{3} \mathrm{NO}_{2}$ moieties. Elementary analysis gave: $\mathrm{C}, 46.2 ; \mathrm{H}, 3.4 ; \mathrm{N}, 15.2$. $\mathrm{C}_{7} \mathrm{H}_{6} \mathrm{~N}_{2} \mathrm{O}_{4}$

Fig. 1. Mass spectrum of G7063-2

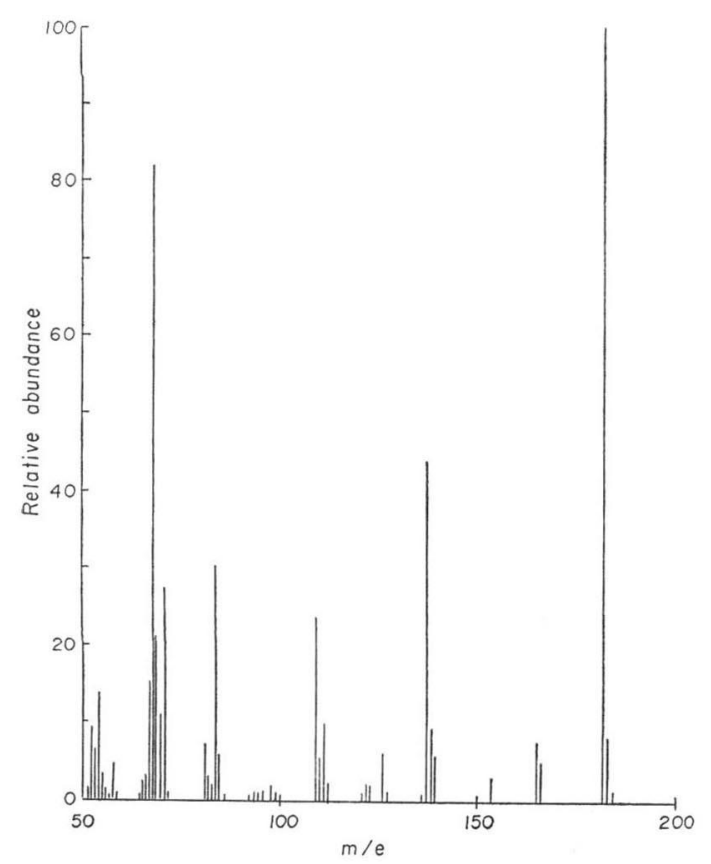

requires $\mathrm{C}, 46.2 ; \mathrm{H}, 3.3 ; \mathrm{N}, 15.4 \%$.

Fig. 2 shows the ultraviolet absorption spectrum of G7063-2 in water and in $0.1 \mathrm{~N} \mathrm{NaOH}$. Maxima (nm with $\mathrm{E}_{1 \mathrm{~cm}}^{1 \%}$ values within brackets) were obtained in water at 248 (430), 292 (740) and 358 (72); $0.1 \mathrm{~N} \mathrm{HCl}, 249$ (450), 292 (690) and 358 (75); $0.1 \mathrm{~N} \mathrm{NaOH}, 249$ sh (870), 260 (1010).

Fig. 2. Ultraviolet spectra of G7063-2

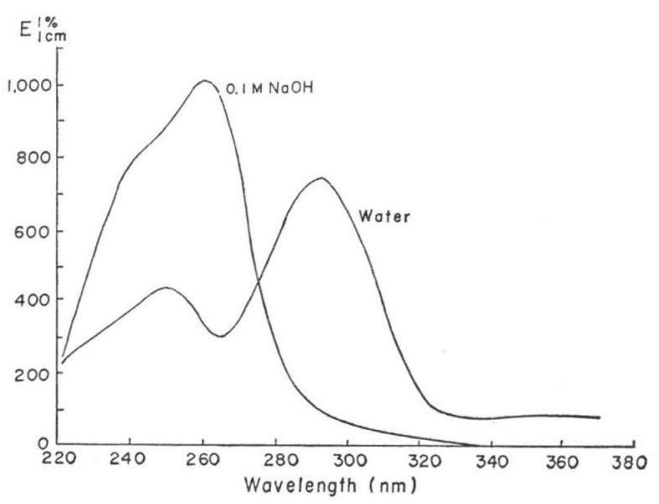


Fig. 3. Infrared spectrum of G7063-2 (Nujol)

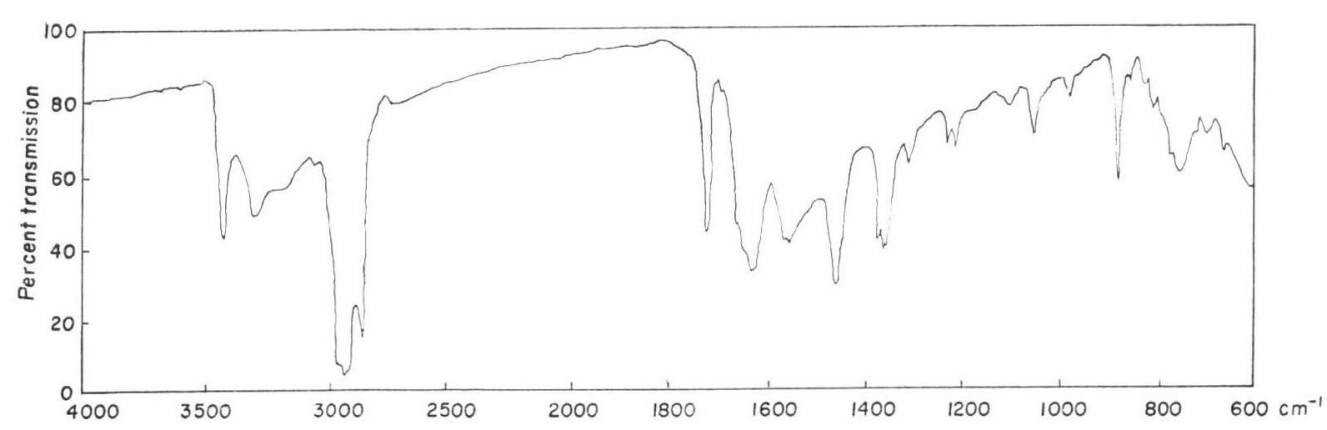

The infrared spectrum of a Nujol mull of G7063-2 (Fig. 3) shows peaks<smiles>NC(=O)C1C(=O)C2OC2C(=O)C1N</smiles>
at 3440 and $3300\left(-\mathrm{NH}_{2}\right)$ and at 1720,1630 and $1555 \mathrm{~cm}^{-1}\left[\mathrm{NH}_{2} \mathrm{CO} \cdot \mathrm{C}(\mathrm{CO} \cdot \mathrm{R})\right.$ $=\mathrm{C} \cdot \mathrm{COR}]$.

A $100 \mathrm{MHz}$ proton NMR spectrum of a solution of G7063-2 in deutero acetone shows signals centred at $\tau 5.92(\mathrm{~d}, 4 \mathrm{~Hz}, 1 \mathrm{H})$ and $6.13(\mathrm{~d}, 4 \mathrm{~Hz}, 1 \mathrm{H})$ for vicinally coupled epoxide protons, at $\tau 1.40$ and $3.42(2 \mathrm{H})$ for amide protons and at $\tau-0.60$ for amine protons $(2 \mathrm{H})$.

The carbon-13 NMR spectrum of a solution of G7063-2 in deuterated dimethylsulphoxide shows seven carbon resonances (attributions within brackets) at $\delta 52.5$ and $55.0(a$ and $b), 97.6(e), 154.7(d)$, $169.4(f)$ and 188.5 and 188.8 ( $c$ and $g$ ) ppm.

Rf values for G7063-2 obtained after thin-layer chromatography on cellulose and silica (see Experimental Section) are given in Table 1.

\section{Biological Properties}

The antibacterial and antifungal spectra are given in Tables 2 and 3.

G7063-2 has moderate activity against a wide range of Gram-positive and Gram-negative bacteria. Against fungi its activity is weak to moderate. Given i. p. to mice the $\mathrm{LD}_{50}$ was about $17 \mathrm{mg} / \mathrm{kg}$ body

Table 1. Rf values of G7063-2 on TLC plates

\begin{tabular}{l|l|l}
\hline \multicolumn{1}{c|}{ Solvent } & Support & \multicolumn{1}{c}{ Rf } \\
\hline Benzene & Silica & 0 \\
Chloroform & & 0.13 \\
Ethyl acetate & & 0.67 \\
Dioxan & & 0.88 \\
Acetone & 0.77 \\
Methanol & 0.74 \\
Butanol-acetic acid-water \\
$\begin{array}{c}\text { (3:1:1) } \\
\text { Ethyl acetate - methanol } \\
\quad(3: 1)\end{array}$ & & 0.61 \\
$\begin{array}{c}\text { Propanol - pyridine - acetic } \\
\text { acid - water } \\
(15: 10: 3: 12)\end{array}$ & Cellulose & 0.84 \\
$\begin{array}{c}\text { Butanol - acetic } \\
\text { acid - water (3:1:1) }\end{array}$ & & \\
$\begin{array}{c}\text { Butanol - methanol - water } \\
(4: 1: 2)\end{array}$ & & 0.70 \\
\hline
\end{tabular}
weight.

\section{Discussion}

After growth of the Streptomyces sp., antibiotic G7063-2 was extracted from the centrifuged broth with ethyl acetate and further purified by chromatography on Sephadex LH20 with two solvent systems. The first system separated G7063-2 from an antibiotic with activity against Staphylococcus aureus but not against E. coli. This substance was shown to have strong activity against Piricularia oryzae and it appeared to be valinomycin ${ }^{3,4)}$ by comparison (TLC, IR spectrum, products of acid hydrolysis and biospectrum) with an authentic sample.

G7063-2 has the same cyclohexenedioneepoxide structure as the antitumour and antibacterial substance terreic acid ${ }^{5)}$, produced by 
Table 2. Antibacterial spectrum of G7063-2

\begin{tabular}{l|c}
\multicolumn{1}{c|}{ Organism* } & MIC $(\mu \mathrm{g} / \mathrm{ml})$ \\
\hline Staphylococcus aureus 663 & 2 \\
Staphylococcus aureus $853 \mathrm{E}$ & 4 \\
Micrococcus sp. $1810 \mathrm{E}$ & 2 \\
Streptococcus faecalis $850 \mathrm{E}$ & 62 \\
Streptococcus pneumoniae 1910E & 62 \\
Streptococcus pyogenes 618 & 16 \\
Bacillus subtilis NCIB 9883 & $<0.5$ \\
Bacillus cereus NCIB 8849 & 8 \\
Haemophilus influenzae 1184E & 4 \\
Escherichia coli $1193 \mathrm{E}$ & 16 \\
Escherichia coli $1507 \mathrm{E}$ & 31 \\
Escherichia coli C1343 & 16 \\
Escherichia coli $1852 \mathrm{E}$ & 8 \\
Klebsiella aerogenes $1082 \mathrm{E}$ & 31 \\
Klebsiella aerogenes $1522 \mathrm{E}$ & 31 \\
Enterobacter cloacae $1051 \mathrm{E}$ & 16 \\
Enterobacter cloacae 1321E & 31 \\
Proteus morganii 235 & 4 \\
Proteus mirabilis $431 \mathrm{E}$ & 16 \\
Pseudomonas aeruginosa 1371E & 125 \\
Serratia marcescens 1324E & 31 \\
\hline
\end{tabular}

* All organisms are Glaxo strains except the Bacillus species.

Table 3. Antifungal spectrum of G7063-2

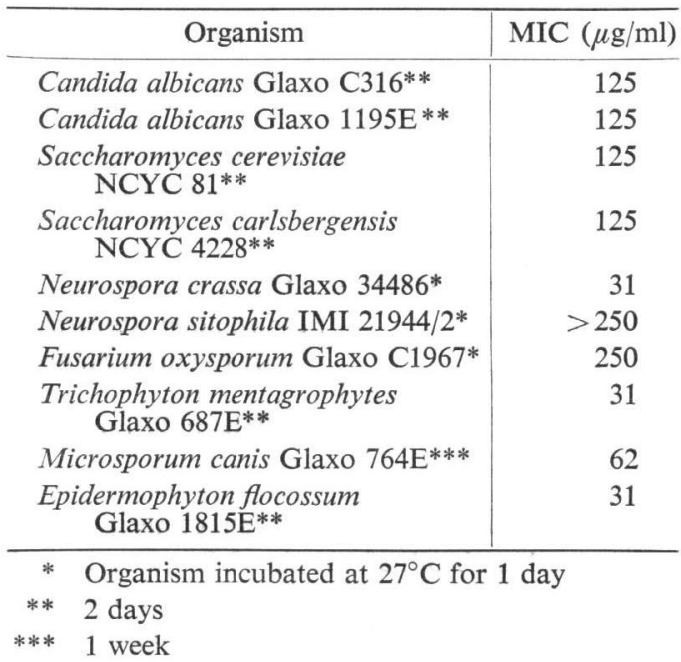

Aspergillus terreus and the phytotoxin, phyllostine $^{6)}$, produced by Phyllosticta sp. However, the stereochemistry about the epoxide group has not been investigated for G7063-2.

The biosynthesis of terreic acid ${ }^{7)}$ and phyllosinol $^{8)}$ (epoxydon) is by the acetate-malonate pathway but whether G7063-2, produced by a Streptomyces sp. and containing nitrogen is synthesised by this route or by the shikimate pathway is not known.

\section{Acknowledgement}

The authors thank Dr. R.A. FLETTON for advice on the interpretation of the NMR spectra; Mr. M.T. DAVIES for the mass spectrum; Patricia Harris, Susan Lobley and Dr. M.E. Bushell for fermentation and Mr.C. Callaghan for extraction of the antibiotic.

\section{References}

1) BERDY, J.: Recent developments of antibiotic research and classification of antibiotics according to chemical structure. Adv. Appl. Microbiol. 18: 309 406, 1974

2) Tippett, L. O.; L. D. Seleznick \& C. A. RobB: Modification of the microliter technique for antimicrobial drug susceptibility testing by incorporation of indicators. Appl. Microbiol. 20: 342 345, 1970

3) Brown, R.; J. Brennan \& C. Kelley: An antifungal agent identical with valinomycin. Antibiot. \& Chemoth. 12: 482 487, 1962

4) Nishimura, H.; M. Mayama, T. Kimura, A. Kimura, Y. Kawamura, K. Tawara, Y. Tanaka, S. Okamoto \& H. Kyotani: Two antibiotics identical with nonactin and valinomycin obtained from a Streptomyces tsusimaensis, n. sp. Taxonomic characteristics of the organism, isolation and characterization, identity with nonactin and valinomycin and antimicrobial properties, especially their specific activity against Piricularia oryzae. J. Antibiotics, Ser. A 17: 11 22, 1964

5) Sheehan, J.; W. Lawson \& R. Gaul: The structure of terreic acid. J. Am. Chem. Soc. 80: 5536, 1958

6) Sakamura, S.; J. Ito \& R. SaKaI: Phyllostine, a new phytotoxic compound produced by Phyllosticta sp. Agr. Biol. Chem. 34: 153 155, 1970

7) Read, G.; D. W. S. Westlake \& L. C. Vining: Quinone epoxides. V. Biosynthesis of terreic acid. Canad. J. Biochem. 47: 1071 1079, 1969

8) Nabeta, K.; A. Ichihara \& S. Sakamura: Biosynthesis of epoxydon and related compounds by Phyllosticta sp. Agr. Biol. Chem. 39: 409 413, 1975 